Improving physical health assessment of old age inpatients on the Oaks Acute Admission Ward

Dr Karen Aus*, Marilia Gougoulaki, Maja Elia and Emily Wall Barnet, Enfield and Haringey Mental Health Trust

${ }^{\star}$ Corresponding author.

doi: 10.1192/bjo.2021.536

Aims. Old age psychiatry patients are subject to increased frailty, comorbid load and medication adverse events than equivalent older age populations without psychiatric illness. Timely physical health assessment and monitoring is therefore an essential part of treatment provision. The Oaks is a 20-bed old age acute admissions ward in Barnet, Enfield and Haringey Mental Health Trust. With this quality improvement project, we aimed to deliver high-quality assessment and treatment of physical health for our patients.

Method. Using NICE guidelines as a blueprint, we devised a list of parameters essential to the management of old age inpatients. This included blood tests (full blood count, urea and electrolytes, liver function, thyroid function, cholesterol, lipids, iron studies, vitamin D, glycated haemoglobin, prolactin), investigations (imaging, ECG, physical examination, cognitive testing) and assessments (body mass index [BMI], functional review, mobility, Rockwood Frailty Score). The implementation goal was to ensure all parameters were acted on within 24 hours of admission (or 48 hours for patients admitted on weekends).

We initially audited these parameters in patients admitted to the Oaks in October and November $2020(n=24)$. We subsequently collated all parameters into an online spreadsheet, which was distributed to ward medical staff. For each new admission, parameters could be marked as pending or complete. The spreadsheet was reviewed in daily ward handover. Following implementation, we collected data on the parameters for patients admitted in December 2020 and January $2021(\mathrm{n}=16)$.

Result. Prior to implementation of the spreadsheet, $42.0 \%$ of all parameters had been actioned within 24 hours of admission. Following the implementation of the spreadsheet, $86.2 \%$ of parameters had been actioned within 24 hours (mean difference $44.2 \%, 95 \%$ CI $13.5 \%$ to $64 \%, \mathrm{p}=0.006$ ).

In detail, there were significant increases in timely actioning of magnesium (increased by $61.7 \%, \mathrm{p}<0.001)$, cholesterol $(61.7 \%$, $\mathrm{p}<0.001)$, glycated haemoglobin $(65.8 \%, \mathrm{p}<0.001)$, vitamin $\mathrm{D}$ $(65.8 \% . \mathrm{p}<0.001)$, prolactin $(61.7 \% \mathrm{p}<0.001)$, lipids $(61.7 \%$, $\mathrm{p}<0.001)$, thyroid function $(51.7 \%, \mathrm{p}<0.01)$, iron studies $(80.9 \%, \mathrm{p}<0.001)$, imaging $(42.5 \%, \mathrm{p}=0.01)$, frailty scores $(60.0 \%, \mathrm{p}<0.01)$, BMI measurement (55.9\%, $\mathrm{p}<0.001)$, and functional review $(42.5 \%, \mathrm{p}=0.01)$.

Conclusion. Implementation of a monitoring spreadsheet with relevant parameters linked to daily ward handover resulted in widespread and significant improvement in the assessment of physical health among old age psychiatry inpatients.

\section{Use of clonidine in the management of opiate withdrawal in community patients}

David Kelsey ${ }^{1 \star}$, Pierre Hoezoo ${ }^{2}$ and Pardeep Grewal ${ }^{3}$

${ }^{1}$ North London Forensic Service; ${ }^{2}$ Enable - Enfield alcohol and drug service and ${ }^{3}$ The Grove Drug Treatment Service

${ }^{\star}$ Corresponding author.

doi: 10.1192/bjo.2021.537

Aims. Clonidine has been used to alleviate symptoms of opiate withdrawal. No validated prescribing schedules exist for the use of Clonidine in opiate detoxification in community patients. We have devised a Clonidine prescribing schedule for adult outpatients seeking opiate detoxification.

Background. Opiate cessation following prolonged use produces a central noradrenergic (NA) response in the locus coeruleus (LC), causing symptoms that can result in reinstatement of use. Pharmacotherapies for withdrawal are thought to work through decreased NA release in the LC by agonising pre-synaptic alpha-2 adrenoceptors. Clonidine has been used since the 1970s. However, it is off-license in the UK, and superseded by Lofexidine. Though both cause hypotension, this is less marked with Lofexidine, which may be anxiolytic and considered better tolerated. Lofexidine is no longer available in the UK. Specialists may need to resort to Clonidine for those seeking opiate detoxification.

Method. We performed a feasibility study with the primary outcome being tolerability of an outpatient clonidine schedule. Patients $(n=7)$ were aged between 18 and 65 years (mean 32). Six were prescribed buprenorphine as opiate substitution (OST), and one methadone.

Exclusion criteria were in keeping with BNF contraindications.

An ECG was obtained for each patient before treatment. A urine drug screen and Clinical Opiate Withdrawal Scale were taken to confirm opiate dependence and withdrawal. Patients selfmonitored withdrawal using the Subjective Opiate Withdrawal Scale and daily blood pressure measurements. Standard adjuvants for withdrawal were prescribed.

A test dose of $100 \mathrm{mcg}$ Clonidine was given to assess for hypotension. If tolerant they received $100 \mathrm{mcg}$ QDS, reducing over eight days.

Patients were contacted by their recovery worker twice during the period.

Result. Five of the seven completed the course, two dropped out due to hypotension. No other adverse effects warranting discontinuation were encountered. Patients reported fatigue and lightheadedness as their most troublesome side-effects. Of 3 patients who returned SOWS scores, 2 reported decline by 21/64 and $14 / 64$ respectively. One reported an increase of 49/64 over 8 days. 3 of the 5 subjects who completed the course were not abstinent at completion, citing opiate withdrawal symptoms as causative.

Conclusion. There is scope for the safe use of clonidine in the community for motivated individuals. Adequate monitoring of heart rate and blood pressure is required. Starting doses at $100 \mathrm{mcg}$ QDS appear well tolerated. Prescribers may wish to reduce this over a longer period to encourage completion and improve tolerability. Further research is needed.

An audit: fitness to drive assessment in inpatients of general adult and old age psychiatry

Ismail Khan ${ }^{1 \star}$, Nneamaka Asiodu ${ }^{2}$, Dr Divyanish ${ }^{3}$, Anum Yaqoob ${ }^{4}$ and Hasanain Qureshi ${ }^{4}$

${ }^{1}$ Bloxwich Hospital; ${ }^{2}$ Bushey Fields Hospital; ${ }^{3}$ Heath Lane hospital and ${ }^{4}$ Dorothy Pattison Hospital

${ }^{\star}$ Corresponding author.

doi: $10.1192 /$ bjo.2021.538

Aims. To determine if fitness to drive is assessed on admission and discharge, if applicable, and for this to be documented during clerking and on discharge notifications.

To determine if patients are being educated about the impact of their condition on the ability to safely drive. 
To ascertain if patients are aware of the duty to inform the DVLA if they for any reason are not fit to drive.

Background. Risk factors include social, behavior and iatrogenic factors such as social withdrawal, increased likelihood of substance abuse and side effects of anti-psychotic medication.

Method. This trust wide audit involved the random sampling of a total of 71 case notes, 4 case notes per Consultant team in general adult psychiatry and old age psychiatry across Dudley and Walsall sites (total of 3 sites). A data collection tool was developed and included relevant questions regarding fitness to drive. Data were collected between October and December 2019.

Result. 18/49 patients had physical health screening prior to medication initiation.

Conclusion. An important aspect of good medical practice is to educate patients about their condition, this includes their fitness to drive as this can be affected both by their diagnosis and medication. It is clear that clinicians also need to be educated about this responsibility to ensure assessment is performed especially on inpatient discharge.

\section{Improving physical health care for inpatients with eating disorders}

Edward Knights*, Monique Schelhase, Rhys Jones and Lou Burke LYPFT NHSFT

${ }^{*}$ Corresponding author.

doi: 10.1192/bjo.2021.539

Aims. Primary aim - To improve how physical health issues are addressed for inpatients with eating disorders

Secondary aim - To improve efficiency within the MDT

Background. The Yorkshire Centre for Eating Disorders (YCED) is an inpatient unit for the treatment of patients with anorexia and bulimia nervosa. Anorexia nervosa has the highest mortality of all psychiatric disorders with an extensive list of physical manifestations. This project was designed to help better address the physical health concerns of our patients by introducing a primary care style, once weekly clinic that patients could self-refer to.

Method. Questionnaires were designed to assess whether a once weekly physical health clinic would benefit the service.

The clinic was run on a weekly basis from 26th April to 24th June 2019. Follow-up questionnaires were designed and distributed to both patients and staff following this period. Data were analysed with Microsoft Excel to determine if improvement had been made.

Result. $\mathrm{N}=12$ inpatients responded to the initial questionnaires, $\mathrm{n}=2$ were discharged during the 8 week period so were included in the analysis but did not complete the follow-up questionnaire.

$100 \%$ of the staff $(n=8)$ felt a once weekly clinic would benefit their patients. $62 \%(n=5)$ stated they felt distracted from their other duties with physical health requests.

$33 \%(n=4)$ of the inpatient group felt the clinic would benefit them with $67 \%(n=8)$ stating indifference to the idea.

26 appointments were conducted in the physical health clinic with $80 \%(n=8)$ of the service users accessing at least once. $70 \%$ $(n=7)$ stated their physical health concerns had been better addressed since the clinic had been started.

90\% ( $n=9)$ of inpatients and 90\% $(n=9)$ of staff responded that the physical health clinic should remain permanent. $90 \%$ $(n=9)$ of staff stated they had more time for their other duties since the introduction of the clinic.

Prior to the clinic 63\% $(n=5)$ of staff responded that in a typical day they were approached between 2-5 times for physical health requests with the other $37 \%(n=3)$ being approached once.

Following the clinic $80 \%(n=8)$ of staff responded that they were approached once in a typical working day.

Conclusion. The qualitative data from the questionnaires indicated success in both improving patient care and reducing nursing workload.

The physical health clinic has been made a permanent feature on the ward and has been continued by the incoming foundation doctor and ward ANP.

\section{Assessement of a structured technological support intervention on uptake of video consultations}

Tejas Kotwal ${ }^{1 \star}$, Kerushan Thomas ${ }^{1}$, Carlos Escudero King ${ }^{1}$ and Avirup Gupta ${ }^{2}$

${ }^{1}$ king's college london and ${ }^{2}$ South London and Maudsley NHS

Foundation Trust

${ }^{*}$ Corresponding author.

doi: 10.1192/bjo.2021.540

Aims. The coronavirus pandemic has led to an increased reliance on remote patient-clinician interactions, mainly the use of telephone and video consultations. Video consultations are key in psychiatric care, as telephone appointments do not sufficiently allow clinicians to accurately ascertain a patient's mental status and perform a risk assessment. The aim of our quality improvement project was to increase the uptake of video consultations within a community mental health team, focusing on substituting telephone consultations for video.

Method. We accessed Electronic Patient Records to retrospectively quantify the method of contact for 130 consultations delivered over a 4-week period. After collecting baseline data, we conducted focused interviews with 10 care providers, identifying the specific clinician and patient barriers to video uptake that informed our intervention design.

Our intervention consisted of two 4-week Plan, Do, Study, Act (PDSA) cycles.

PDSA 1 involved delivering a focused PowerPoint presentation to the care team, highlighting the benefits of video consultation technology and encouraging clinicians to use it as their primary method of remote communication with patients. Additionally, we conducted qualitative interviews with members of the team to highlight the successes and challenges thus far.

PDSA 2 involved creating a video consultation instructional PDF which highlighted how to operate the technological aspects of both Microsoft Teams and WhatsApp Video Call. This included: how to set-up video calls, accept invitations, and overcome common troubleshooting issues.

The proportion of remote consultations was quantified retrospectively to compare trends in video consultation uptake from baseline to the conclusion of PDSA 2.

Result. Overall, we saw a 15\% increase in video consultations with respect to baseline. The greatest change was attributable to PDSA cycle 1 , which incurred an $8 \%$ increase in video consultation uptake, from $13.85 \%$ to $21.9 \%$. PDSA cycle 2 further increased video consultation uptake by $6.97 \%$, from $21.9 \%$ to $28.87 \%$. Specifically focusing on remote consultations, the proportion conducted with video rather than telephone increased by $17.3 \%$. Interviewed clinicians reported limited financial access, technological fluency, and issues with patient privacy as the most important barriers to the uptake of video consultations.

Conclusion. Our project successfully increased the proportion of consultations conducted by video. This was achieved by targeting 